\section{Vision-Based Grasp Tracking for Planar Objects}

Gabriel Recatalá, Member, IEEE, Raffaella Carloni, Member, IEEE, Claudio Melchiorri, Member, IEEE, Pedro J. Sanz, Member, IEEE, Enric Cervera, Member, IEEE, and Ángel P. del Pobil, Member, IEEE

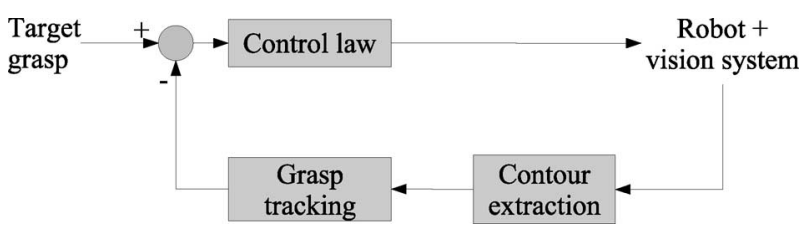

Fig. 1. Grasp-based positioning movement.
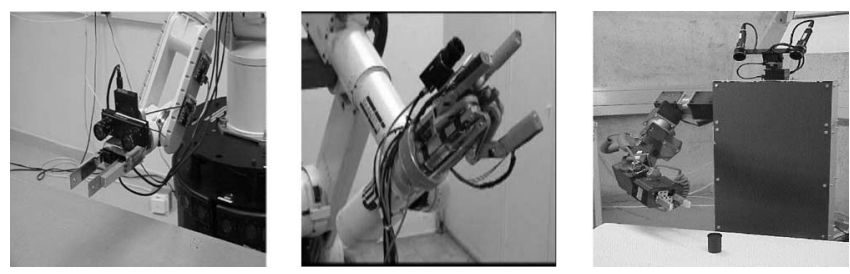

Fig. 2. Some experimental setups for tracking a grasp.

tasks, we base the visual servoing control loop on the tracking of those points. We compute a stable grasp at the beginning of the task, and then, we transfer it along the sequence of acquired images. This has two main advantages: first, the computational cost of each iteration of the control loop is reduced; and second, by avoiding the grasp search at each iteration, the errors due to noisy sensor data decrease.

The proposed procedure, depicted in Fig. 1, requires three functional blocks: the extraction of a set of features in the images (i.e., the contour of the object to be grasped), the selection of a stable grasp configuration and its tracking along different views of the object, and the control law whose target is the grasp configuration. In particular, this correspondence paper focuses on the design of the grasp tracking module, which allows both to transfer the grasp points along a sequence of images and to find matches between pairs of images. The purpose of this module is to be used as a tool within a visual servoing system to control the positioning of a robot with respect to a set of grasp points selected on an object. This is specially useful in robots that have to perform precision manipulation tasks.

In this correspondence paper, the vision system is considered uncalibrated, so that the internal camera parameters are not known or used. The object is not known a priori and no model of it is available; it is assumed to be rigid and planar: ideally, it is a shape that lies on a given plane, so it is considered relatively flat. The concepts presented in this correspondence paper have been applied in three different setups, shown in Fig. 2: the Robotic Intelligence Laboratory at the University Jaume I in Castellón, the Laboratory of Automation and Robotics at the University of Bologna, and the Laboratory for Process Control and Real Time Systems at the Technical University of Munich.

\section{GRASP TRACKING}

The grasp tracking module of Fig. 1 can be subdivided as shown in Fig. 3. During the approaching movement of the robotic arm toward the object to be grasped, a sequence of contours is acquired in real time by the vision system. At the beginning of this sequence, as no grasp is available, an initial stable grasp is computed in the first contours (grasp synthesis) [10], [12], [13]. Then, for each new incoming contour, the algorithm tries to apply a previously computed grasp to the current image. This process is called here grasp transfer. This involves a transfer of that grasp configuration to the current image and an evaluation of the stability of the transferred grasp, i.e., a grasp analysis [14]. 


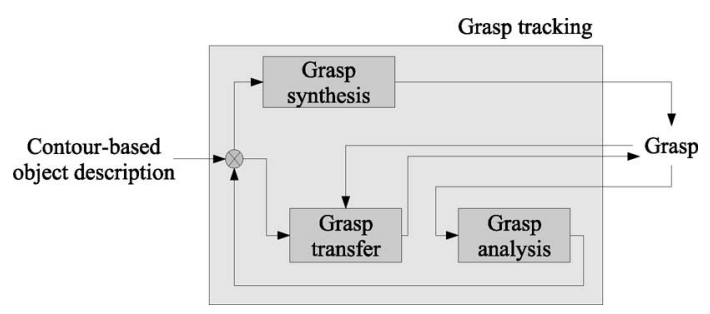

Fig. 3. Grasp tracking procedure.

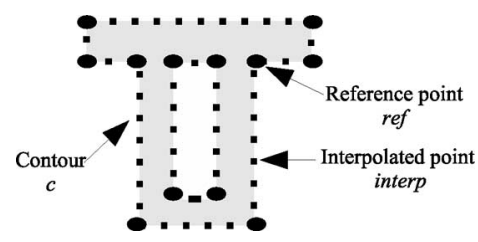

Fig. 4. RRP coordinates of contour points.

The procedures for the grasp synthesis and analysis have already been presented elsewhere [10], [12] and are out of the scope of this paper. This correspondence paper focuses on three different options for the grasp transfer strategy, described in Section IV. Section III presents the grasp description each of these strategies is based on, using it as an invariant for the grasp transfer, and explains the context in which such a description, and therefore, its associated grasp transfer strategy, can be used.

\section{INVARIANTS FOR GRASP TRANSFER}

\section{A. Grasp-Related Invariant Features: RRP Coordinates}

In the representation using the relative reference points $(R R P)$ coordinates, the contour is considered as a polygon. The vertices of this polygon are named reference points and the contour points that lie on the segment joining the reference points are the interpolated points. Therefore, the RRP coordinates for the generic $i$ th grasp point consists of three items: the index $c$ of the contour inside the image view; the index ref of the reference point, within the list of reference points corresponding to contour $c$; and the index interp of the point, interpolated between the reference point $r e f$ and the following one. Fig. 4 depicts the RRP coordinates.

In this case, the invariance cannot be ensured unless the whole set of contour points is rigidly transferred from one image to the next one. Thus, the tracking of the grasp points has to be performed within the tracking of the entire object. It follows that, since the object tracking method [10] has been developed for images that are correlated by translations and rotations along the normal direction to the image plane, the movements of the robotic arm are bound to these 4 DoFs.

\section{B. Grasp-Related Invariant Features: Grasp-Line Coordinates}

The description with the grasp-line coordinates is only for antipodal grasps and is based on the specification of the relative location of the grasp line (the line joining the two grasp points) with respect to a reference frame in which the axes are the minimum $\left(I_{\mathrm{m} i n}\right)$ and maximum $\left(I_{\max }\right)$ inertia axes of the silhouette of the object and the center is the centroid $\left(p_{c}\right)$. As depicted in Fig. 5, this description is composed of the following:

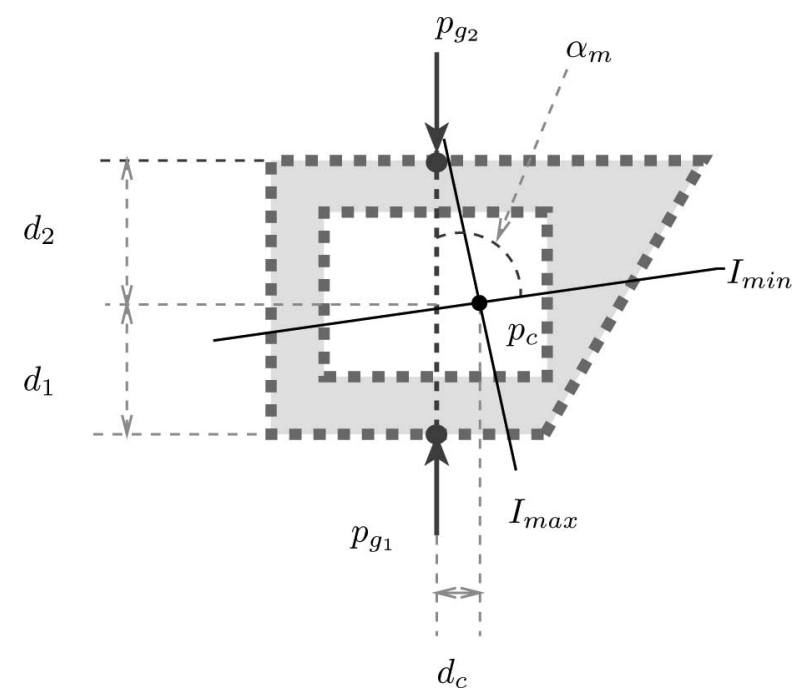

Fig. 5. Grasp description using grasp-line coordinates.

TABLE I

GRASP DESCRIPTIONS IN RRP AND GRASP-LINE COORDINATES

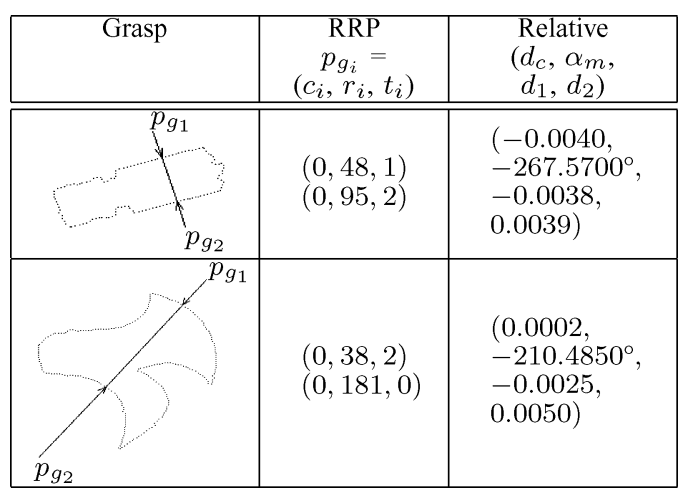

1) $d_{c}$ : distance between the grasp line and the centroid $p_{c}$;

2) $\alpha_{m}$ : angle between the grasp line and the $I_{\mathrm{m} \text { in }}$ axis, measured from $I_{\mathrm{m} \text { in }}$ toward the grasp line;

3) $d_{i}$ : distance, along the direction vector of the grasp line, between a grasp point, $p_{g_{i}}$, and the intersection between the grasp line and $I_{\min }(i \in\{1,2\})$.

This description is based on the computation of second-order normalized moments of the object silhouette, which are invariant under 4 DoFs of movements of the vision system with respect to the image plane [15]. The invariance of the grasp-line coordinates requires that the whole object can be observed in each image. Partial occlusions of the shape of the object or enlargements would cause the loss of the invariance of the geometric moments between pairs of images, so that this description of the grasp could not be used. Table I shows grasp descriptions expressed in RRP and grasp-line coordinates.

\section{Grasp-Related Invariant Features: Homography}

This grasp description is based on the computation of a linear projective transformation between two object views, a homography [16]. This transformation produces, given a point in a plane, its corresponding point in the other plane. In general, a projective transformation between two projective planes $I$ and $I^{\prime}$ can be represented by a general 


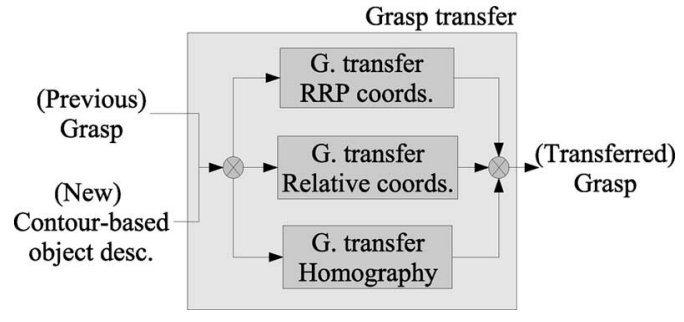

Fig. 6. Grasp transfer strategies.

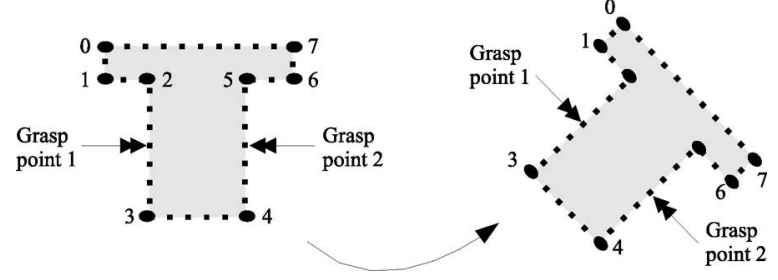

Fig. 7. Invariance of the RRP coordinates. With a contour index 0 , the RRP coordinates of the grasp points in both contours are $(0,2,4)$ and $(0,4,4)$.

linear transformation in projective space

$$
\left[\begin{array}{l}
x_{1}^{\prime} \\
x_{2}^{\prime} \\
x_{3}^{\prime}
\end{array}\right]=\mathbb{H}\left[\begin{array}{l}
x_{1} \\
x_{2} \\
x_{3}
\end{array}\right] \quad \mathbb{H}=\left[\begin{array}{lll}
h_{11} & h_{12} & h_{13} \\
h_{21} & h_{22} & h_{23} \\
h_{31} & h_{32} & h_{33}
\end{array}\right]
$$

with $\mathbf{x}=\left[\begin{array}{lll}x_{1} & x_{2} & x_{3}\end{array}\right]^{T} \in I, \mathbf{x}^{\prime}=\left[\begin{array}{lll}x_{1}^{\prime} & x_{2}^{\prime} & x_{3}^{\prime}\end{array}\right]^{T} \in I^{\prime}$. Equation (1) can then be rewritten as

$$
\left[\begin{array}{c}
\lambda_{i} u_{i}^{\prime} \\
\lambda_{i} v_{i}^{\prime} \\
\lambda_{i}
\end{array}\right]=\mathbb{H}\left[\begin{array}{c}
u_{i} \\
v_{i} \\
1
\end{array}\right], \quad i \in[1, \ldots, 4]
$$

where $\lambda_{i} \in \mathbb{R}$ is a scalar, $\mathbf{p}=\left[\begin{array}{ll}u & v\end{array}\right]^{T}$ and $\mathbf{p}^{\prime}=\left[\begin{array}{ll}u^{\prime} & v^{\prime}\end{array}\right]^{T}$ are the coordinates in Cartesian space of $\mathbf{x}$ and $\mathbf{x}^{\prime}$, respectively. They can be obtained as $u=x_{1} / x_{3}, v=x_{2} / x_{3}, u^{\prime}=x_{1}^{\prime} / x_{3}^{\prime}$, and $v^{\prime}=x_{2}^{\prime} / x_{3}^{\prime}$. The homography matrix is invariant with respect to a 6 DoFs movement between two object views.

\section{GRASP TRANSFER}

As depicted in Fig. 6, the grasp-transfer block proposes three options for this operation. Each of them is based on one of the invariants described in Section III.

\section{A. Grasp Transfer Based on RRP Coordinates}

Following the specifications given in Section III-A, this strategy is applied together with an object-tracking algorithm, described in [10], based on the use of B-splines as active contours [17]. In particular, in the contour tracking, the points sampled from the B-spline provide the RRP coordinates, i.e., the reference and interpolated points. Fig. 7 shows the application of this transfer strategy.

\section{B. Grasp Transfer Based on Grasp-Line Coordinates}

The grasp-line coordinates of the grasp points provide a description $\left(d_{c}, \alpha_{m}, d_{1}, d_{2}\right)$. As the grasp is initially expressed in the first object as a pair of points, those points are used to compute these coordinates. They are then applied to the second object and used to compute the position of the grasp points in that object, as shown in Fig. 8.

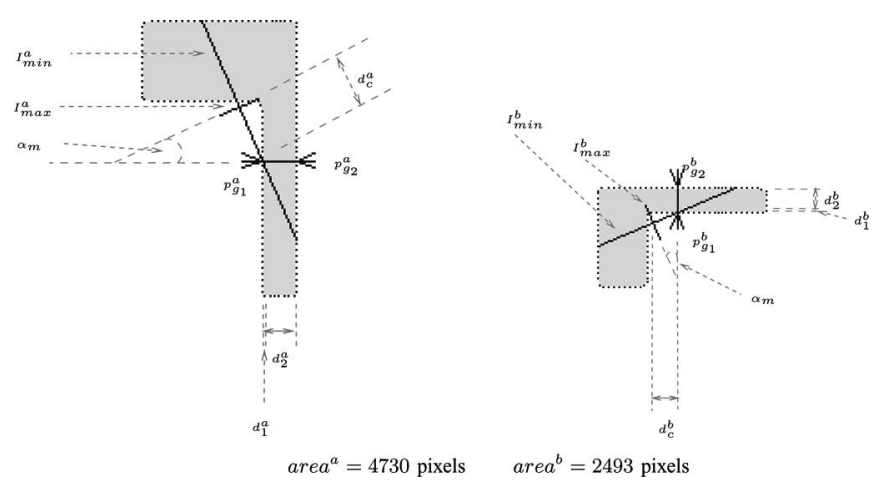

Fig. 8. Invariance of the grasp description based on grasp-line coordinates. The grasp is described by $d_{c}=0.0060, \alpha_{m}=-66.8701^{\circ}, d_{1}=-0.0001$, $d_{2}=0.0036$, and $d_{i}=d_{i}^{j} /$ area $^{j}$.

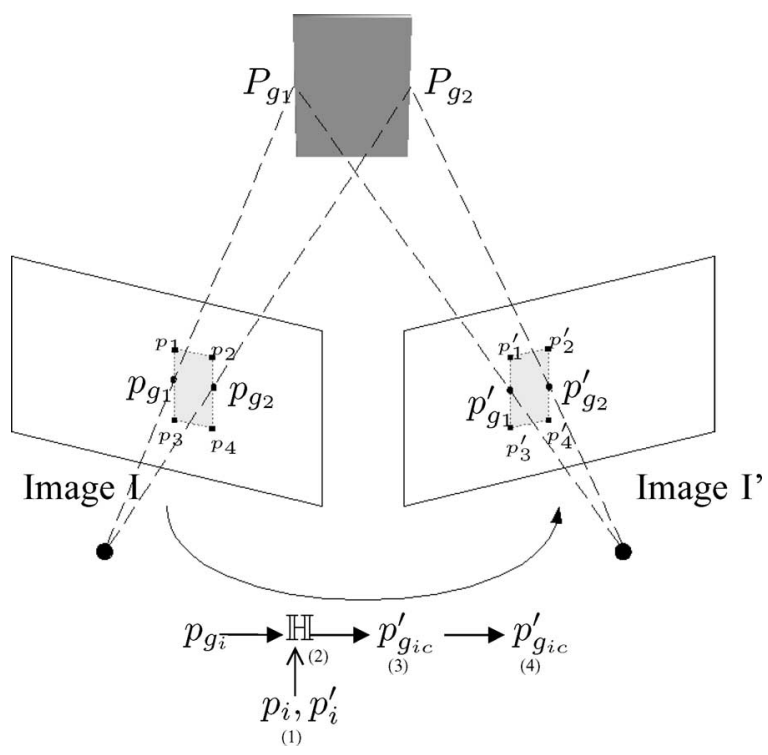

Fig. 9. Four-step use of a homography for the transfer of grasp points.

\section{Grasp Transfer Based on a Homography}

In this strategy, a homography is used for transferring the grasp points. In this case, it is necessary to find at least four correspondences, provided that no three of them are collinear, between two projectively transformed planes to define the homography matrix $\mathbb{H}$ uniquely [18]. As shown in Fig. 9, the proposed procedure involves four steps: 1) search of correspondences; 2) computation of the homography; 3 ) application of the homography; and 4) grasp refinement.

1) Search of Correspondences: The search of correspondences between two views of the same scene is a typical problem of computer vision [19]-[21]. In most cases, a manual selection is required in the first view or there must exist some specific relationship between both views.

The proposed procedure can be described as follows. First, as four correspondences are required, four points are selected in the contour of one of the objects. For this purpose, the curvature of each contour is analyzed to select the four peaks with the highest curvature [18].

Next, the procedure tries to match those points on the other contour. For each point $\mathbf{p}_{\mathbf{i}}$ from the first contour, it tries to find the match between the curvature vectors of both contours, as shown in Fig. 10. This search is performed within an interval $\left[i_{c}-\kappa \xi, i_{c}+\kappa \xi\right]$, where $\kappa=1$ in the first contour and a scale factor in the second one, computed as the ratio between the lengths $l_{c}$ and $l_{c}^{\prime}$ of both contours, and $\xi$ is the radius of this interval in the first contour. Let $\mathbf{p}_{\mathbf{j}_{\mathrm{i}}}^{\prime}$ be a point of the 

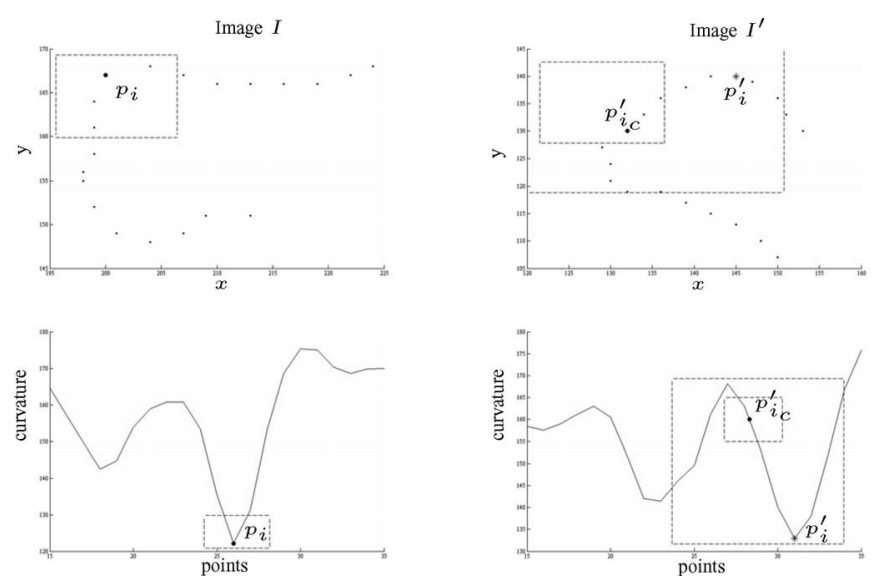

Fig. 10. Search of the correspondence of a given point.

TABLE II

SELECTED POINTS $(*)$ AND CONTOUR CURVATURE ON THE FIRST CONTOUR AND THEIR CORRESPONDENCES $(\bullet)$

First/second contour

second contour; the matching error $d\left(\mathbf{p}_{\mathbf{i}}, \mathbf{p}_{\mathbf{j}_{\mathbf{i}}}^{\prime}\right)$ is

$$
d\left(\mathbf{p}_{\mathbf{i}}, \mathbf{p}_{\mathbf{j}_{\mathbf{i}}}^{\prime}\right)=\sum_{k=-n}^{n}\left|v(i+k)-v^{\prime}\left(j_{i}+\kappa k\right)\right|
$$

where $v(x)$ and $v^{\prime}(x)$ are the values of the $x$ th element of the curvature vector of the first and the second contours, respectively, and $n$ is the radius of the neighborhood considered for comparing both curvature vectors. The matching neighborhood is considered to be that with the lowest matching error. The center $\mathbf{p}_{\mathbf{i}}^{\prime}$ of such neighborhood is taken as the point correspondence of $\mathbf{p}_{\mathbf{i}}$. Table II shows the correspondences $\mathbf{p}_{\mathbf{i}}^{\prime}$ $(\bullet)$ in the second contour of the points $\mathbf{p}_{\mathbf{i}}(*)(i \in[1, \ldots, 4])$ selected in the first contour.

This procedure takes advantage of the fact that the object contours from consecutive frames, taken within short time periods, are similar to each other. This allows the matching of correspondences be performed individually point to point. For more different images (i.e., with faster object movement or more ellapsed in time), a more complex, global matching procedure would be required.

2) Computation of the Homography: Let the four corresponding points be $\left[\begin{array}{ll}u_{1} & v_{1}\end{array}\right]^{T},\left[\begin{array}{ll}u_{2} & v_{2}\end{array}\right]^{T},\left[\begin{array}{ll}u_{3} & v_{3}\end{array}\right]^{T},\left[\begin{array}{ll}u_{4} & v_{4}\end{array}\right]^{T}$, in plane $I$ and $\left[\begin{array}{ll}u_{1}^{\prime} & v_{1}^{\prime}\end{array}\right]^{T},\left[\begin{array}{ll}u_{2}^{\prime} & v_{2}^{\prime}\end{array}\right]^{T},\left[\begin{array}{ll}u_{3}^{\prime} & v_{3}^{\prime}\end{array}\right]^{T},\left[\begin{array}{ll}u_{4}^{\prime} & v_{4}^{\prime}\end{array}\right]^{T}$ in plane $I^{\prime}$. The substitution of these points in (2) produces a linear system that can be used to compute the components of $\mathbb{H}$.

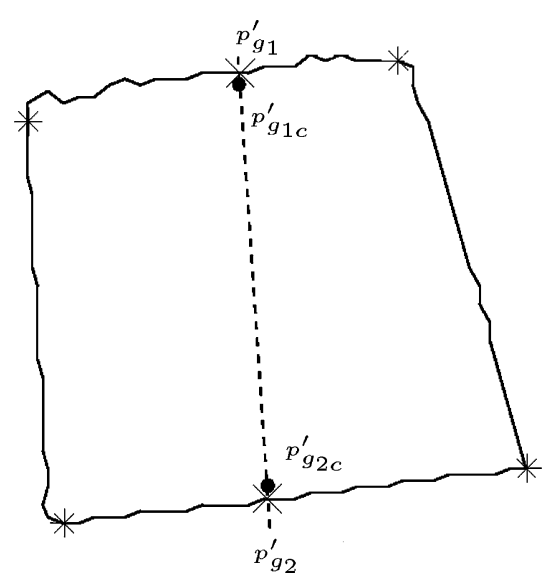

Fig. 11. Grasp refinement. Correction of the position of the grasp points.

TABLE III

GRASP TRANSFER BASED ON RRP COORDINATES

\begin{tabular}{|c|c|c|c|}
\hline $\begin{array}{c}\text { Original/transferred } \\
\text { grasp }\end{array}$ & $\begin{array}{c}\text { Location } \\
p_{g_{i}}= \\
\left(c_{i}, p_{i}\right)\end{array}$ & $\begin{array}{c}\text { RRP } \\
p_{g_{i}}= \\
\left(c_{i}, r_{i}, t_{i}\right)\end{array}$ & $\begin{array}{c}\text { Relative } \\
\left(d_{c}, \alpha_{m},\right. \\
\left.d_{1}, d_{2}\right)\end{array}$ \\
\hline & & & \\
& $(0,587)$ & $(0,40,6)$ & -9.0001, \\
& $(0,242)$ & $(0,15,25)$ & -0.0013, \\
& & & $0.0007)$ \\
& & & \\
& $(0,607)$ & $(0,40,6)$ & $-87.8033^{\circ}$, \\
& $(0,243)$ & $(0,15,25)$ & -0.0012, \\
& & & $0.0007)$ \\
\hline$p_{p_{g_{2}}^{\prime}}$ & & & \\
\hline
\end{tabular}

3) Application of the Homography: Once the homography matrix has been computed, the grasp points $\mathbf{p}_{\mathbf{g}_{\mathrm{i}}}$ from the first contour, available in image $I$, are transferred to image $I^{\prime}$, producing points $\mathbf{p}_{\mathbf{g}_{\text {ic }}}^{\prime}$ in this image.

4) Grasp Refinement: Ideally, the points $\mathbf{p}_{\mathbf{g}_{\text {ic }}}^{\prime}$ should lie on the corresponding contour in $I^{\prime}$. The correction in their position, so that they really lie on the contour, can be achieved by using known restrictions in the relationship between the grasp points. Fig. 11 shows the case in which these points have been computed for a two-finger gripper: the line joining the two grasp points is considered to be the transferred grasp line and the intersections between this line and the contour are computed. At least, there should be two intersections. The two intersections $\mathbf{p}_{\mathbf{g}_{1}}^{\prime}$ and $\mathbf{p}_{\mathbf{g}_{2}}^{\prime}$ that are closest to the transferred points are considered to be the grasp points in image $I^{\prime}$.

\section{RESUlts}

In this section, we analyze the results of the proposed transfer methods, their error, and their computational cost. The experimental setup considered for testing the grasp transfer is described in Section I. The object contours used in the tests are the input to the block grasp transfer shown in Fig. 3, and are extracted from camera-acquired images [10], [18].

Tables III-V show some examples of grasp transfer based on RRP and grasp-line coordinates, and on a homography, respectively. In Table III, the sampled points of a B-spline are used to set the reference points. As it can be observed in Tables III and IV, the coordinates used as invariants for the grasp transfer remain the same, while the others 
TABLE IV

GRASP TRANSFER USING GRASP-LINE COORDINATES

\begin{tabular}{|c|c|c|c|}
\hline $\begin{array}{c}\text { Original/transferred } \\
\text { grasp }\end{array}$ & $\begin{array}{c}\text { Location } \\
p_{g_{i}}= \\
\left(c_{i}, p_{i}\right) \\
\end{array}$ & $\begin{array}{c}\text { RRP } \\
p_{g_{i}}= \\
\left(c_{i}, r_{i}, t_{i}\right)\end{array}$ & $\begin{array}{c}\text { Relative } \\
\left(d_{c}, \alpha_{m},\right. \\
\left.d_{1}, d_{2}\right)\end{array}$ \\
\hline & $\begin{array}{l}(0,152) \\
(0,296)\end{array}$ & $\begin{array}{l}(0,25,2) \\
(0,49,2)\end{array}$ & $\begin{array}{l}(0.0009, \\
-89.9220^{\circ}, \\
-0.0041, \\
0.0035)\end{array}$ \\
\hline & $\begin{array}{l}(0,172) \\
(0,347)\end{array}$ & $\begin{array}{l}(0,28,4) \\
(0,57,5)\end{array}$ & $\begin{array}{l}(0.0009, \\
-89.9220^{\circ}, \\
-0.0041, \\
0.0035)\end{array}$ \\
\hline
\end{tabular}

TABLE V

GRASP TRANSFER BASED ON A HOMOGRAPHY

\begin{tabular}{|c|c|}
\hline Original grasp & Translated grasp \\
\hline $\begin{array}{l}p_{g_{1}} \vdots \\
\\
\\
\text { Image coordinates } \\
\text { Selected correspondences: } \\
\text { (200, 183) } \\
(253,163) \\
(187,118) \\
(183,169) \\
\text { Selected grasp: } \\
p_{g_{1}}=(187,152) \\
p_{g_{2}}=(201,161)\end{array}$ & $\begin{array}{l}\text { } \\
\end{array}$ \\
\hline
\end{tabular}

change. Table V shows the correspondences used for computing the homography, the result of applying it on the original grasp, and the transferred grasp produced after the refinement step.

The original and the transferred grasps in grasp-line and RRP transfers are similar. The differences are due to discretization errors during the sampling or the extraction of the contour and the computation of the features with respect to which the grasp is described. However, they are lower in the RRP-based transfer, thanks to the use of an object tracking algorithm, which better preserves the shape of the object. With respect to the homography-based transfer, the results show that it is sensitive to the errors in the search of the correspondences for computing the homography; this highlights the importance of the refinement step as an error-compensating method.

For the analysis of the error of the proposed procedures, although they ensure that the transferred grasp points lie on the object contour, we have considered several error measures to evaluate the precision of the proposed techniques in ensuring that the original and the transferred grasp are the same. Let a grasp be defined by points $p_{g_{i}}(i \in\{1,2\})$ in image $I$. Using one of the proposed grasp transfer procedures, we obtain
TABLE VI

REVERSibILITy ERroR $\left(R_{v} E\right)$ OF THE PROPOSED TECHNIQUES

\begin{tabular}{|c|c|c|c|c|}
\hline Original grasps & & RRP & $\begin{array}{c}\text { Grasp-line } \\
\text { Coord. }\end{array}$ & Hom. \\
\hline \hline \multirow{2}{*}{$\begin{array}{c}\text { Figures } \\
\text { in Table III }\end{array}$} & $e_{a v}$ & 0 & 0 & 0 \\
\cline { 2 - 5 } & $e_{\max }$ & 0 & 0 & 0 \\
\hline \multirow{2}{*}{$\begin{array}{c}\text { Figures } \\
\text { in Table IV }\end{array}$} & $e_{a v}$ & 0 & 0.00024 & 0.00063 \\
\cline { 2 - 5 } & $e_{\max }$ & 0 & 0.00049 & 0.00077 \\
\hline \multirow{2}{*}{$\begin{array}{c}\text { Figures } \\
\text { in Table V }\end{array}$} & $e_{a v}$ & 0 & 0 & 0 \\
\cline { 2 - 5 } & $e_{\max }$ & 0 & 0 & 0 \\
\hline
\end{tabular}

TABLE VII

REFERENCE $\left(R_{f} E\right)$ AND SEQUENCE ERRORS (SES) OF THE PROPOSED TECHNIQUES

\begin{tabular}{|c|c|c|c|c|}
\hline Original grasps & & RRP & $\begin{array}{c}\text { Grasp-line } \\
\text { Coord. }\end{array}$ & Hom. \\
\hline & $\begin{array}{l}R_{f} E\left(e_{a v}\right) \\
R_{f} E\left(e_{\max }\right. \\
S E\left(e_{a v}\right) \\
S E\left(e_{\max }\right)\end{array}$ & $\begin{array}{l}0.00026 \\
0.00053 \\
0.00301 \\
0.00322\end{array}$ & $\begin{array}{l}0.00026 \\
0.00053 \\
0.00143 \\
0.00233\end{array}$ & $\begin{array}{l}0 \\
0 \\
0 \\
0\end{array}$ \\
\hline & $\begin{array}{l}R_{f} E\left(e_{a v}\right) \\
R_{f} E\left(e_{\max }\right. \\
S E\left(e_{a v}\right) \\
S E\left(e_{\max }\right)\end{array}$ & $\begin{array}{c}0 \\
0 \\
0.00013 \\
0.00094\end{array}$ & $\begin{array}{c}0 \\
0 \\
0.00036 \\
0.00117\end{array}$ & $\begin{array}{l}0 \\
0 \\
0 \\
0\end{array}$ \\
\hline & $\begin{array}{l}R_{f} E\left(e_{a v}\right) \\
R_{f} E\left(e_{\max }\right. \\
S E\left(e_{a v}\right) \\
S E\left(e_{\max }\right)\end{array}$ & $\begin{array}{l}0.00104 \\
0.00124 \\
0.00561 \\
0.00612\end{array}$ & $\begin{array}{l}0.00850 \\
0.00870 \\
0.00499 \\
0.00545\end{array}$ & $\begin{array}{l}0 \\
0 \\
0 \\
0\end{array}$ \\
\hline
\end{tabular}

grasp points $p_{g_{i}}^{\prime}$ in image $I^{\prime}$. Let dist $t_{i}$ the Euclidean distance between either $p_{g_{i}}$ or $p_{g_{i}}^{\prime}$ and a given reference grasp point, and area $_{o b j}$ the area of the object. In this case, the average error $\left(e_{\mathrm{av}}\right)$ and the maximum error $\left(e_{\max }\right)$ can be defined as

$$
e_{\mathrm{av}}=\frac{\sum_{i=1}^{2} \text { dist }_{i} / 2}{\text { area }_{o b j}} \quad e_{\mathrm{max}}=\frac{\max _{i \in\{1,2\}} \text { dist }_{i}}{\text { area }_{o b j}} .
$$

As dist $_{i}$ is measured in pixels and area $_{o b j}$ in square pixels, both in image space, $e_{\mathrm{av}}$ and $e_{\mathrm{max}}$ are provided in 1/pixels. The proposed error measures are the following.

1) Reversibility error $\left(R_{v} E\right)$ : Computed using a pair of images. Let $p_{g_{i}}^{\prime \prime}$ be the result of back-transferring $p_{g_{i}}^{\prime}$ to image $I$. dist $t_{i}$ is computed in $I$ between $p_{g_{i}}$ and $p_{g_{i}}^{\prime \prime}$.

2) Reference error $\left(R_{f} E\right)$ : Computed between pairs of images. dist $_{i}$ is computed in $I^{\prime}$ between $p_{g_{i}}^{\prime}$ and a point $p_{g_{i}}^{r}$ known to be the actual correspondence of $p_{g_{i}}$ in $I^{\prime}$.

3) Sequence error (SE): Computed along a sequence of images. It is the reference error along the sequence. It is computed as the average $e_{\mathrm{av}}$ and the maximum $e_{\mathrm{max}}$ along the sequence. It is intended to provide a statistical estimation of the stability of the grasp tracking.

Table VI shows the reversibility error computed on some examples from previous figures and tables. For the reference and the sequence errors (SEs), shown in Table VII, we have used synthetic contours along a predefined trajectory. In this way, the homography can reconstruct this synthetic trajectory, and we obtain a ground truth with respect to which we can compare the results of the proposed transfer procedures. The SE has been computed over a sequence of 100 frames, and the reference error on frame 50 of this sequence. Note that the SE statistically highlights the differences between the three grasp transfer techniques. 
As it can be observed in the earlier tables, the homography-based transfer provides a reduced error if the movement of the object can be recovered through the homography matrix, while the RRP-based transfer can also be accurate when the shape of the object is not modified. These two methods can also handle a wider variety of types of grasps than the grasp-line-based transfer and other grasp-tracking methods [22]. Anyway, the tests for the SE were based on synthetic data, providing a more favorable situation for the homography-based method. Nevertheless, the errors obtained with the three methods are minimal. However, as each transfer strategy is based on a grasp description defined in a specific context (see Section III), when selecting such a strategy, it is necessary to consider other issues too, such as time constraints, the object description, and the relative movement of the object.

Finally, regarding the computational cost of the proposed grasp transfer methods, those based on RRP and grasp-line coordinates are the fastest, with computation times, respectively, below 15 and $35 \mu \mathrm{s}$, for a two-finger gripper and contours of around 300 points on a $2.5-\mathrm{GHz}$ Pentium Celeron. The homography-based method is slower, requiring $290 \mathrm{~ns}$ under the same configuration, with $60 \%$ of the time spent in search of correspondences. The grasp analysis, performed together with the transfer during tracking, adds only $370 \mu$ s. The total time is, in all cases, below $1 \mathrm{~ms}$.

\section{CONCLUSION}

In this correspondence paper, a method for the tracking of grasp points in image space has been described. Among other applications, this method enables the use of grasp points as control features within a visual servoing loop [14], [18]; it avoids repeating the grasp search at each iteration of the loop and ensures that the same grasp is considered during the positioning movement. It can also be integrated within the manipulation of objects in 3-D space, being useful for the tracking of a given grasp (possibly selected with more complex criteria than in the 2-D case) along a sequence of 2-D images acquired during exploration or approximation movements with respect to the object.

The method proposed in this correspondence paper is really interesting in practical applications, as shown in the results. Each transfer strategy has shown some advantages and disadvantages. In particular, the RRP-based grasp transfer requires the use of an object-tracking method, but it benefits from a more stable description of the object with respect to which the grasp is located. The grasp transfer using grasp-line coordinates does not rely on the tracking of the object, but is quite dependent on the full observability of the contour of the object, as well as of the robustness of the contour-extraction method.

Finally, homography-based transfer has the advantages of allowing the tracking of a variable number of grasp points and, with a careful selection of the point correspondences, of not necessarily requiring a view of the whole shape of the object. Nevertheless, its computation is more complex than that of the other methods.

\section{REFERENCES}

[1] F. Berry, P. Martinet, and J. Gallice, "Real time visual servoing around a complex object," IEICE Trans. Inf. Syst., vol. E83-D, no. 7, pp. 13581368, Jul. 2000 .
[2] E. Malis, F. Chaumette, and S. Boudet, "Multi-cameras visual servoing," in Proc. IEEE Int. Conf. Robot. Autom., vol. 4, San Francisco, CA, Apr.2000, pp. 3183-3188.

[3] P. Li, F. Chaumette, and O. Tahri, "A shape tracking algorithm for visual servoing," in Proc. IEEE Int. Conf. Robot. Autom., Barcelona, Spain, Apr. 2005, pp. 2847-2852.

[4] R. Horaud, F. Dornaika, and B. Espiau, "Visually guided object grasping," IEEE Trans. Robot. Autom., vol. 14, no. 4, pp. 525-532, Aug. 1998.

[5] P. Allen, A. Timcenko, B. Yoshimi, and P. Michelman, "Automatic tracking and grasping of a moving object with a robotic hand-eye system," IEEE Trans. Robot. Autom., vol. 9, no. 2, pp. 152-165, Apr. 1993.

[6] J. Zhang, Q. J. Wu, H.-T. Tsui, and W. Gruver, "Binocular transfer methods for point-feature tracking of image sequences," IEEE Trans. Syst., Man Cybern., Part C, vol. 32, no. 4, pp. 392-405, Nov. 2002.

[7] M. Moussa and M. Kamel, "An experimental approach to robotic grasping using a connectionist architecture and generic grasping functions," IEEE Trans. Syst., Man, Cybern., Part C, vol. 28, no. 2, pp. 239-253, May 1998.

[8] E. Chinellato, A. Morales, R. Fisher, and A. del Pobil, "Visual features for characterizing robot grasp quality," IEEE Trans. Syst., Man Cybern., Part C, vol. 35, no. 1, pp. 30-41, Feb. 2005.

[9] K. Stanley, Q. J. Wu, and W. Gruver, "Implementation of vision-based planar grasp planning," IEEE Trans. Syst., Man Cybern., Part C, vol. 30, no. 4, pp. 517-524, Nov. 2000.

[10] G. Recatalá, M. Sorg, J. Leupold, P. Sanz, and A. del Pobil, "Visual grasp determination and tracking in 2D dynamic scenarios," in Proc. IEEE/RSJ Int. Conf. Intell. Robots Syst., Lausanne, Switzerland, Oct.2002, pp. 2530.

[11] C. Davidson and A. Blake, "Error-tolerant visual planning of planar grasp," in Proc. 6th Int. Conf. Comput. Vision, Bombay, India, 1998, pp. 911-916.

[12] A. Macchelli, C. Melchiorri, R. Carloni, and M. Guidetti, "Space robotics: An experimental set-up based on RTAI-linux," presented at the 4th RealTime Linux Workshop, Boston, MA, Dec. 2002.

[13] J. Cornellà and R. Suárez, "Fast and flexible determination of force-closure independent regions to grasp polygonal objects," in Proc. IEEE Int. Conf. Robot. Autom., Barcelona, Spain, Apr. 2005, pp. 778-783.

[14] G. Recatalá, P. Sanz, E. Cervera, and A. del Pobil, "Grasp-based visual servoing for gripper-to-object positioning," in Proc. IEEE/RSJ Int. Conf. Intell. Robots Syst., Sendai, Japan, Sep. 2004, pp. 118-123.

[15] R. González and P. Wintz, Digital Image Processing, 2nd ed. Reading, MA: Addison-Wesley, 1987.

[16] R. Hartley and A. Zisserman, Multiple View Geometry in Computer Vision, 2nd ed. Cambridge, U.K.: Cambridge Univ. Press, 2004.

[17] A. Blake and M. Isard, Active Contours. New York: Springer-Verlag, 1998.

[18] R. Carloni, G. Recatalá, C. Melchiorri, P. Sanz, and E. Cervera, "Homography-based grasp tracking for planar objects," in Proc. IEEE Int. Conf. Robot. Autom., New Orleans, LA, Apr. 2004, pp. 795-800.

[19] Z. Zhang, R. Deriche, O. Faugeras, and Q.-T. Luong, "A robust technique for matching two uncalibrated images through the recovery of the unknown epipolar geometry,” INRIA, Sophia-Antipolis, France, Tech. Rep. 2273 May 1994.

[20] P. Mendonça, K.-Y. Wong, and R. Cipolla, "Epipolar geometry from profiles under circular motion," IEEE Trans. Pattern Anal. Mach. Intell., vol. 23, no. 6, pp. 604-616, Jun. 2001.

[21] G. Chesi, E. Malis, and R. Cipolla, "Collineation estimation from two unmatched views of an unknown planar contour for visual servoing," in Proc. Brit. Mach. Vision Conf., Nottingham, U.K., Sep. 1999, pp. 224 233.

[22] T. Mishra, P. Guha, A. Dutta, and K. Venkatesh, "Efficient continous regrasp planning for moving and deforming planar objects," in Proc. IEEE Int. Conf. Robot. Autom., Orlando, FL, May 2006, pp. 2472-2477. 